\title{
Cosmological black holes as seeds of voids in the galaxy distribution
}

\author{
S. Capozziello ${ }^{1,4}$, M. Funaro ${ }^{2}$, and C. Stornaiolo ${ }^{3,4}$ \\ 1 Dipartimento di Fisica "E.R. Caianiello", Università di Salerno, via S. Allende, 84081 Baronissi (Salerno), Italy \\ 2 Dipartimento di Matematica e Informatica, Università di Salerno, via Ponte Don Melillo, 84084 Fisciano (Salerno), Italy \\ 3 Dipartimento di Scienze Fisiche, Università di Napoli, Complesso Universitario di Monte S. Angelo, via Cinthia, Edificio \\ N - 80126 Napoli, Italy \\ ${ }^{4}$ Istituto Nazionale di Fisica Nucleare, Sezione di Napoli, Complesso Universitario di Monte S. Angelo, via Cinthia, \\ Edificio G, 80126 Napoli, Italy
}

Receveid 5 February 2004 / Accepted 11 March 2004

\begin{abstract}
Deep surveys indicate a bubbly structure on cosmological large scales which should be the result of evolution of primordial density perturbations. Several models have been proposed to explain the origin and dynamics of such features but, till now, no exhaustive and fully consistent theory has been found. We discuss a model where cosmological black holes, deriving from primordial perturbations, are the seeds for large-scale-structure voids. We give details of the dynamics and accretion of the system voids-cosmological black holes from the epoch $z \simeq 10^{3}$ till now, finding that a void of $40 \mathrm{~h}^{-1} \mathrm{Mpc}$ diameter and under-density of -0.9 fits the observations without conflicting with the homogeneity and isotropy of the cosmic microwave background radiation.
\end{abstract}

Key words. cosmology: large scale structure of Universe - cosmology: dark matter - black hole physics

\section{Introduction}

The existence of voids has been evident after the discovery by Kirshner et al. 1981 of a large void with a diameter of $60 \mathrm{Mpc}$ in Böotes. Systematic surveys have shown the existence of many regions with similar characteristics. Computer analysis of galaxy distribution gives evidence that voids occupy about $50 \%$ of the volume of the universe (e.g., see El-Ad \& Piran 1997) or, according to a more recent paper (Hoyle \& Vogeley 2002), about $40 \%$ of the volume of the universe.

Today, there is a general agreement on the issue that voids are not just empty regions of space, but that they are regions with a very low density of luminous matter.

As observed by Peebles (2001), the velocity dispersion of galaxies indicates that most matter must be inside the voids, not only if the density parameter (for the matter component) $\Omega_{m}=1$ but also for $\Omega_{m} \ll 1$. In any case, recent observations (de Bernardis et al. 2000; Perlmutter et al. 1999; Schimdt et al. 1998) indicate that the total value of the density parameter is $\Omega=\Omega_{m}+\Omega_{\Lambda}=1$ where $\Omega_{m} \simeq 0.3$ and $\Omega_{\Lambda} \simeq 0.7$. Here $\Omega_{\Lambda}$ is the contribution due to the whole content of unclustered matter which can be the cosmological constant, some kind of scalar field (Caldwell et al. 1998; de Ritis et al. 2000;

Send offprint requests to: S. Capozziello, e-mail: capozziello@sa.infn.it
Rubano \& Barrow 2001; Capozziello 2002) or, in general, "dark energy".

It is worthwhile to stress that the visual inspection of the galaxy distribution suggests nothing other than the absence of large amounts of luminous matter in wide regions. Furthermore, it is not clear whether the voids are spherical regions approximately empty or under-dense regions with arbitrary shapes. Several definitions of voids have been proposed, but a general agreement on their real nature has not been reached yet (Schmidt et al. 2001).

The Swiss-Cheese cosmological model, initially proposed by Einstein \& Straus (1945, 1946), appears suitable for the description of the cosmological voids. In a recent paper (Stornaiolo 2002), it was proposed to approach the formation of the cosmological voids in the framework of this model. It was shown that voids are the consequence of the collapse of extremely large wavelength perturbations into low-density black holes and of the comoving expansion of matter surrounding the collapsed perturbations.

As a result, it was claimed that in the center of each void there is a black hole whose mass $M$ compensates the mass which the void would have if it were completely filled with matter having a cosmological density.

In Stornaiolo (2002) the voids are empty regions of the universe which grow in a comoving frame with the cosmological expansion. In that paper, the presence of cosmic background radiation was neglected. 
In this paper, we analyze the physical mechanism capable of explaining the structure of voids in the presence of baryonic matter and cosmic background radiation (CBR) with central black holes acting as seeds. The layout of the paper is the following: in Sect. 2, we will present the cosmological black hole $(\mathrm{CBH})$ model in the framework of the Friedmann-Lemaître-Robertson-Walker (FLRW) cosmology. Section 3 is devoted to the discussion of the effects of interaction between the CBR and a CBH. A mechanism for the formation of an under-dense regime void is analyzed in Sect. 4, while the comparison with observations, which makes it possible to determine the initial time of voids formation and the mass function of CBHs, is studied in Sect. 5. The discussion of the results and the conclusions are given in Sect. 6.

\section{The CBH model and Cosmology}

The cosmological model proposed in Stornaiolo (2002) is an Einstein-Straus universe which is embedded in an FLRW metric. A central spherical black hole with mass

$M=\frac{4}{3} \pi \Omega_{\mathrm{CBH}} \rho_{\mathrm{c}} R_{v}^{3}$,

is present in all the voids.

In Eq. (1), the parameter

$\Omega_{\mathrm{CBH}}=\frac{\rho_{\mathrm{CBH}}}{\rho_{\mathrm{c}}}$

represents the fraction of density due to all these black holes with respect to the total density of the universe; $\rho_{\mathrm{c}}=1.88 \times$ $10^{-29} \mathrm{~g} \mathrm{~cm}^{-3} h^{2} \Omega$ is the present-day critical density of the universe.

All the voids are assumed to be spherical.

A black hole forms when a body of mass $M$ collapses entirely within a sphere of radius

$R_{\mathrm{s}}=\frac{2 G M}{c^{2}}$

This statement is equivalent to say that its density satisfies the relation,

$R_{\mathrm{s}}(\rho)=\sqrt{\frac{3 c^{2}}{8 \pi G \rho}}$.

Conversely, Eq. (4) defines for any density a corresponding Schwarzschild radius. In other words, any space-like sphere of matter, with uniform density $\rho$ and radius equal to $R_{\mathrm{s}}(\rho)$, is a black hole.

From Eqs. (1) and (3), we determine the mass $M$ and the corresponding Schwarzschild radius and consequently, from Eq. (4) the mass density of the central black hole. For example, a black hole in the center of a $50 \mathrm{~h}^{-1} \mathrm{Mpc}$ diameter void would have a mass $1.8 \times 10^{16} M_{\odot}$ corresponding to a Schwarzschild radius $1.7 \mathrm{kpc}$ and a density of $2.34 \times 10^{-16} \mathrm{~g} \mathrm{~cm}^{-3}$.

The above value of the density is the one reached by the collapsing matter when it crossed the Schwarzschild radius. It suggests that the process of formation of a $\mathrm{CBH}$ started with large wavelength perturbations at cosmological densities of the order of $10^{-19} \mathrm{~g} / \mathrm{cm}^{3}$ i.e. for $1+z \approx 10^{3}$. According to the inflationary scenario, we only need to suppose that the inflation occurred during a time long enough to provide such perturbations ${ }^{1}$.

Note that, since the Einstein-Straus model assumes spherical symmetry, the perturbation does not experience the cosmic expansion during its collapse.

For this reason, we can assume that the total mass of the perturbation

$M_{\mathrm{p}}=\frac{\pi}{6} \Omega_{\mathrm{p}} \rho_{i} \lambda_{i}^{3}$

remains constant during the whole process.

The Schwarzschild radius of the spherical perturbation is equal to

$R_{\mathrm{s}}=\frac{H_{i}^{2}}{c^{2}}\left(\frac{\lambda_{i}}{2}\right)^{3} \Omega_{\mathrm{p}}$

We can distinguish two cases:

1. the case in which the relation

$\frac{2 R_{\mathrm{s}}}{\lambda_{i}} \geq 1$,

is satisfied. The perturbation is in the linear regime and, according to the evolution equations of a universe with perfect fluid equation of state, it is frozen when $\lambda_{i}$ is larger than the Hubble radius (Mukhanov et al. 1992). After crossing the Hubble horizon, it collapses and becomes a black hole when

$\left(\frac{\lambda_{i}}{2}\right)^{2} \geq \frac{c^{2}}{H_{i}^{2} \Omega_{\mathrm{p}}}$.

2. The case where

$\frac{2 R_{\mathrm{s}}}{\lambda_{i}}<1$.

Here the perturbation evolves as shown in Stornaiolo (2002). During the contraction, it becomes unavoidably a black hole if the final density is very low and the internal pressure and temperature cannot increase to values large enough to prevent the collapse. This is true also when the perturbation enters a non-linear regime. In addition, any possible centrifugal barrier can be reduced to values smaller than the Schwarzschild radius (Loeb 1993) by the interaction of collapsing matter with the cosmic background radiation. According to the Einstein-Straus model, after the formation of the black hole the matter around it expands in a comoving way leading to the formation of an empty region between it and the rest of the universe. As the central black hole cannot be seen, the whole region appears as a void to an external observer.

$\mathrm{A} \mathrm{CBH}$ can be detected through its lensing properties, since it behaves like a Schwarzschild gravitational lens. According to our hypothesis, a CBH sits in the center of a void and the Einstein angle is (Schneider et al. 1992)

$\alpha_{0}=4.727 \times 10^{-4} \Omega_{\mathrm{CBH}}^{1 / 2} \sqrt{\frac{R_{V}^{3} D_{d \mathrm{~s}}}{D_{d} D_{\mathrm{s}}}}$,

1 The Oppenheimer-Snyder model (Oppenheimer \& Snyder 1939) describes the spherical symmetric collapse at zero pressure. 
where $R_{V}$ is the radius of the void, $D_{\mathrm{s}}$ is the distance of the source from the observer, $D_{d \mathrm{~s}}$ is the distance of the source from the $\mathrm{CBH}$, and $D_{d}$ is the distance of the $\mathrm{CBH}$ from the observer; all these quantities are expressed in Mpc. For a $50 \mathrm{Mpc}$ void, with the center placed at a distance of $80 \mathrm{Mpc}$ from the Sun and with a source at the opposite edge of the void, we expect a deflection angle

$\alpha_{0} \simeq 3.2 \times 10^{-4} \Omega_{\mathrm{CBH}}^{1 / 2}$.

On the other hand, Zeldovich \& Sazhin (1987) point out that generally static structures can raise the temperature of the cosmic background radiation by an amount proportional to the Hubble parameter and the gravitational time delay. Considering the Swiss-Cheese model case, they find a temperature fluctuation $\delta T / T \sim 10^{-10}$ for a giant galaxy $\left(M=4 \times 10^{12} M_{\odot}\right)$. Since this result is proportional to the mass, it follows that it corresponds to a fluctuation of temperature $\delta T / T \sim 10^{-5}$ for a large $\mathrm{CBH}$ with mass $M \sim 10^{17} M_{\odot}$, which does not contradict the recent Boomerang and WMAP measurements (de Bernardis et al. 2000; Tegmark et al. 2003).

An interesting feature of voids with central $\mathrm{CBH}$ is derived starting from a Swiss-Cheese model based on an Einsteinde Sitter cosmology. To this aim, let us consider the energy balance in Newtonian terms for a galaxy with mass $m$ sitting on the edge of a void i.e. at a distance $r=r_{e} a(t)$ where $r_{e}$ is a covariant radius. We have

$E=\frac{1}{2} m v^{2}-G \frac{m M_{v}}{r_{e} a}$.

As the void is expanding in a comoving frame, we can impose $v=H r_{e} a(t)$. The mass $M_{v}$ inside the void is

$M_{v}=\frac{4 \pi \rho_{\mathrm{int}}\left(r_{e} a\right)^{3}}{3}$.

By Eq. (11), one obtains

$E=\frac{m\left(r_{e} a\right)^{2}}{2}\left[H^{2}-\frac{8 \pi G \rho_{\text {int }}}{3}\right]$

and then

$E=\frac{m\left(r_{e} a\right)^{2}}{2}\left[\frac{8 \pi G}{3}\left(\rho_{\mathrm{tot}}-\rho_{\mathrm{int}}\right)\right]$.

From this relation, we see that, $E=0$ if $\rho_{\text {tot }}=\rho_{\text {int }}$, i.e. the expansion velocity of a galaxy on the edge of a void coincides with the escape velocity ${ }^{2}$. On the other hand, a galaxy on the edge of a void is gravitationally bound to a $\mathrm{CBH}$, if $\rho_{\text {int }}>\rho_{\text {tot }}$. In this paper, we shall adopt as definition of edge of a void the spherical region where the galaxies have energy $E=0$.

These considerations can be immediately extended to the case in which the spatial curvature is different from zero (i.e. to Friedmann models different from the considered Einstein-de Sitter one). This simply implies that

$$
H^{2}=\frac{8 \pi G}{3} \rho_{\text {tot }}-\frac{K}{a^{2}}
$$

\footnotetext{
${ }^{2}$ In the densities $\rho_{\text {int }}$ and $\rho_{\text {tot }}$, we are taking into account all the contributions to the energy density such as matter and radiation.
}

and Eq. (13) becomes

$$
E=\frac{m\left(r_{e} a\right)^{2}}{2}\left[\frac{8 \pi G}{3}\left(\rho_{\mathrm{tot}}-\rho_{\text {int }}\right)-\frac{K}{a^{2}}\right] .
$$

In this case, the value of $E$ depends also on $K / a^{2}$.

\section{Interaction between $\mathrm{CBR}$ and $\mathrm{CBH}$}

So far we have considered the Einstein-Straus Swiss-cheese model in a universe filled only with matter. In this section, we shall show how the interaction with the CBR will lead to accretion by a $\mathrm{CBH}$. Since the pressure of radiation is different from zero, the radiation itself may cross the edge of the voids regardless of the Einstein-Straus junction conditions (Einstein \& Straus 1945, 1946). As we consider this physical process during the matter epoch, we can neglect the contribution of radiation to the black hole formation.

A CBH absorbs energy from the CBR according to the law (Custodio \& Horvath 2002)

$\frac{\mathrm{d} M}{\mathrm{~d} t}=\sigma_{\mathrm{g}}(M) F_{\mathrm{rad}}$

where $\sigma_{\mathrm{g}}(M)=\left(27 \pi G^{2} / c^{4}\right) M^{2}$ is the gravitational cross section of $\mathrm{CBH}$ and $F_{\text {rad }}=\rho_{\mathrm{rad}} c$ is the radiation flux of CBR.

In a matter dominated universe, (dust), the evolution of radiation density is given by

$\dot{\rho}_{\mathrm{rad}}=-3 H\left(\rho_{\mathrm{rad}}+p_{\mathrm{rad}}\right)$

where $H=\frac{2}{3 t}$ and the equation of state is $p_{\text {rad }}=\frac{1}{3} \rho_{\text {rad }}$.

Immediately, we get

$\rho_{\mathrm{rad}}(t)=\frac{A}{t^{\frac{8}{3}}}$,

where the constant $A$ has the dimensions $\left[\mathrm{g} \mathrm{cm}^{-3} \mathrm{~s}^{8 / 3}\right]$. At the present epoch, assuming $t_{0} \cong 3.08 \times 10^{17} h^{-1} \mathrm{~s}$ (this value is consistent with WMAP observations (Tegmark et al. 2003) for $h \cong 0.7)$ and $\rho_{\text {rad }}\left(t_{0}\right) \cong 4.8 \times 10^{-34} \mathrm{~g} \mathrm{~cm}^{-3}$, we obtain

$\rho_{\text {rad }}(t) \cong \frac{2.08 \times 10^{13} h^{-8 / 3}}{t^{\frac{8}{3}}}$,

disregarding the physical dimensions.

Equation (17) becomes

$\frac{\mathrm{d} M}{\mathrm{~d} t}=\frac{2.94 \times 10^{-31} h^{-8 / 3} M^{2}}{t^{\frac{8}{3}}}$.

Integrating from an initial epoch $t_{i}$ to the present epoch, we have that the ratio between the present mass $M_{0}$ and the initial mass $M_{i}$ is

$M_{0}=\frac{M_{i}}{1+1.76 \times 10^{-31} h^{-8 / 3} M_{i}\left(\frac{1}{t_{0}^{5 / 3}}-\frac{1}{t_{i}^{5 / 3}}\right)}$

If $t_{i} \ll t_{0}$, we can approximate the formula for the mass accretion to

$M_{0}=\frac{M_{i}}{1-\frac{1.76 \times 10^{-31} h^{-8 / 3} M_{i}}{t_{i}^{5 / 3}}}$. 
The growth of mass of a $\mathrm{CBH}$ is then

$$
\begin{aligned}
\Delta M & =M_{0}-M_{i} \\
& =M_{i}\left[\frac{1.76 \times 10^{-31} h^{-8 / 3} M_{i}\left(\frac{1}{t_{0}^{5 / 3}}-\frac{1}{t_{i}^{5 / 3}}\right)}{1+1.76 \times 10^{-31} h^{-8 / 3} M_{i}\left(\frac{1}{t_{0}^{5 / 3}}-\frac{1}{t_{i}^{5 / 3}}\right)}\right] .
\end{aligned}
$$

For $t_{i} \ll t_{0}$, we have

$\frac{M_{0}}{M_{i}} \simeq 1+\frac{1.76 \times 10^{-31} h^{-8 / 3} M_{i}}{t_{i}^{5 / 3}}$.

Therefore, it is evident that the $\mathrm{BH}$ accretion is proportional to its initial mass.

\section{Under-density inside the voids}

Voids are under-dense regions of space depleted of galaxies with respect to the external background. We can estimate the amount of such an under-density in the framework of our $\mathrm{CBH}-$ void model.

Taking into account the accretion process in the void, we cannot neglect the fact that, as the void increases in volume, a certain amount of galaxies enters in the void contributing to the total mass inside the void. This implies that the accretion of mass inside the void, given by Eq. (17), has to be corrected to

$\frac{\mathrm{d} M}{\mathrm{~d} t}=2 \sigma_{\mathrm{g}}(M) F_{\mathrm{rad}}$

The factor 2 can be justified in the following way.

If we consider any mass increment in Eq. (11), it is easy to observe that the mass of galaxies entering the void is equal to the mass increment of the black hole, if one takes into account the conservation equation for $\rho_{\text {tot }}$. In a first approximation, one can double the righthand side of Eq. (17). To be rigorous, one should consider a delay effect, since gravity does not propagate instantaneously. Due to this, it would be correct to deal with this problem within the standard of the General Relativity. However, it is possible to show that the delay effect can be neglected, at least in first approximation.

To find the density of galaxies $\rho_{\text {und }}$ which enter expanding voids, one has to subtract the accreted mass of the $\mathrm{CBH}$, obtained from Eq. (23), from the total final accretion mass, given by Eq. (26), (in the approximation $t_{i} \ll t_{0}$ )

$$
M_{f}=\frac{M_{i}}{1-3.53 \times 10^{-31} h-8 / 3 M_{i} t_{i}^{-5 / 3}},
$$

and divide the result by the volume of the void taken as $M_{f} / \rho_{\text {tot }}$. Finally, we find that

$\rho_{\mathrm{und}}=\left(1-\frac{M_{0}}{M_{f}}\right) \rho_{\mathrm{tot}}$

The under-density is given by the contrast of the density of galaxies in the void with the total density $\rho_{\text {tot }}$, i.e.

$\delta=\frac{\rho_{\text {und }}}{\rho_{\text {tot }}}-1=-\left(1+\frac{9.69 \times 10^{16} h^{-2 / 3} V}{t_{i}^{5 / 3}}\right)^{-1}$, where $V$ is the volume of the void expressed in $10^{3} \mathrm{Mpc}^{3}$ and $t_{i}$ is the initial time expressed in seconds. From this last equation, we obtain the formula for the initial time $t_{i}$, which is

$t_{i}=\left(-\frac{\delta}{1+\delta} \times 9.69 \times 10^{16} h^{-2 / 3} V\right)^{\frac{3}{5}}$,

where $\delta$ and $V$ can be retrieved from the respective values found in the catalogs (El-Ad \& Piran 1997). On the other hand, we can also determine the initial masses of the CBHs just after their formation. This can be expressed by the formula

$M_{i}=\frac{\rho_{\mathrm{int}} V}{1-2 \frac{1+\delta}{\delta}}$

where $\rho_{\text {int }}$ is the internal density of the void.

\section{Comparison with the observations}

Using the data of the volume and the under-density of voids given in the catalog of El-Ad \& Piran (1997) which deduced their data from SSRS2 (da Costa et al. 1988, 1994) and IRAS (Fisher et al. 1995) observations, we found the following initial times and corresponding initial and final masses according to formulas $(30,31,27)$.

The dynamics deduced from our model is consistent with the observations and seems to confirm the gravitational origin of voids. In this approach, the role of dark matter has to be revised since most of the mass (about one half) of the structure is concentred in the central $\mathrm{CBH}$. In this picture, it is only the density contrast between voids and background which drives the dynamics. CBHs are just the remnant of primordial collapsed perturbations while voids, or precisely the edges of voids, are the result of perturbations of which the wavelength follows the cosmic expansion. The whole system, also if expanding and interacting with $\mathrm{CBR}$, remains in equilibrium.

\section{Discussion and conclusions}

In this paper, we have developed a model where cosmological black holes are seeds for large scale structure voids. Such systems are a result of the evolution of primordial perturbations and become stable structures from $\left(z \simeq 10^{3}\right)$ up to now. They enlarge to diameters of about $40 \mathrm{~h}^{-1} \mathrm{Mpc}$ and the under-density of voids is of the order -0.9 with respect to the background. The whole structure is a sort of honeycomb where most galaxies (i.e. luminous matter) are located on the edge of the voids while most dynamical mass is sited in the central black hole. The edge is defined by a natural equilibrium condition for the energy due to the balance of the gravitational pull of the central black hole and the cosmic expansion. The cosmic background radiation contributes to the accretion of the black hole but its homogeneity and isotropy are not affected, in agreement with the data. The picture which emerges agrees with optical and IRAS observations (El-Ad \& Piran 1997) giving a 50\% of the volume filled by voids with the above characteristics. The presence of central black holes seems to confirm the gravitational 
Table 1. Evolution of the properties of the voids and black holes features derived from our model using the data in El-Ad \& Piran (1997).

\begin{tabular}{ccccc}
\hline \hline $\begin{array}{c}\text { Volume } \\
10^{3} \mathrm{Mpc}^{3}\end{array}$ & $\begin{array}{c}\text { under-density } \\
\delta\end{array}$ & $\begin{array}{c}\text { initial time } \\
h^{-2 / 5} \times 10^{12} \mathrm{~s}\end{array}$ & $\begin{array}{c}\text { initial CBH masses } \\
\Omega h^{2} \times 10^{16} M_{\odot}\end{array}$ & $\begin{array}{c}\text { final CBH masses } \\
\Omega h^{2} \times 10^{16} M_{\odot}\end{array}$ \\
\hline 84.9 & -0.89 & 0.78 & 1.90 & 2.11 \\
93.9 & -0.87 & 0.74 & 2.01 & 2.28 \\
119.0 & -0.93 & 1.29 & 2.88 & 3.08 \\
24.0 & -0.91 & 0.42 & 0.56 & 0.61 \\
22.6 & -0.94 & 0.53 & 0.56 & 0.59 \\
17.4 & -0.92 & 0.37 & 0.41 & 0.47 \\
8.8 & -0.91 & 0.23 & 0.20 & 0.22 \\
11.4 & -0.95 & 0.39 & 0.29 & 0.30 \\
31.1 & -0.86 & 0.36 & 0.65 & 0.74 \\
22.4 & -0.69 & 0.16 & 0.33 & 0.43 \\
41.5 & -0.88 & 0.48 & 0.91 & 1.02 \\
8.8 & -0.97 & 0.46 & 0.23 & 0.24 \\
10.7 & -0.74 & 0.12 & 0.17 & 0.22 \\
\hline
\end{tabular}

origin of the voids and stabilizes the system against cosmic expansion preventing its evaporation.

It is interesting to note that the order of magnitude observed for the masses of $\mathrm{CBH}$ concides with that of the Great Attractor (Fairall 1997). It is very tempting to identify the Great Attractor as a $\mathrm{CBH}$ and to use the model described in this paper to explain the large scale motions observed for the galaxies surrounding it.

However, the main problem with observations of cosmic velocity fields (Faber et al. 1994) is that the voids are, in general, the opposite to the Great Attractor, and large scale structure around the voids does not show velocity fields converging toward the voids, but toward the visible clusters and superclusters around the voids. This apparent shortcoming, in the framework of our model (see also Stornaiolo 2002), can be overcome by the Birkoff theorem which states that the stationary solutions are also static if the spherical symmetry is restored. So, a fraction of galaxies is attracted by clusters and superclusters "outside" the void while another fraction shows no dynamics since it has been already attracted "inside" the void. This fact could be interpreted as an early selection due to a competitive mechanism between CBHs and external matter contained in clusters and superclusters.

However, if the Swiss-Cheese model were always valid such a selection would never have been achieved; instead, in a more realistic situation, the model holds only approximately so then we have to expect galaxies inside and outside the void due to the deviations from sphericity and to the perturbations of the $\mathrm{CBH}$ mass.

Furthermore, as observed in Davis \& Peebles (1983) and Peebles (2001), the small relative velocity dispersion shows that, if $\Omega_{M}=1$, then most of the mass has to be contained in the voids. The same authors conjecture that this must be true even when $\Omega_{M}$ is smaller than 1 as predicted by several CDM simulations.

\section{References}

Caldwell, R. R., Dave, R., \& Steinhardt, P. J. 1998, Phys. Rev. Lett., 80,1582

Capozziello, S. 2002, Int. J. Mod. Phys. D, 11, 483

Custodio, P. S., \& Horvath, J. E. 2002, GRG, 34, 1895

da Costa, L. N., Pellegrini, P. S., Sargent, W. L. W., et al. 1988, ApJ, 327,544

da Costa, L. N., Geller, M. S., Pellegrini, P. S., et al. 1994, ApJ, 424, L1

Davis, M., \& Peebles, P. J. E. 1983, ApJ, 267, 465

de Bernardis, P., Ade, P. A. R., Bock, J. J., et al. 2000, Nature, 404, 955

de Ritis, R., Marino, A. A., Rubano, C., \& Scudellaro, P. 2000, Phys. Rev. D, 62, 043506

Einstein, A., \& Straus, E. G. 1945, Rev. Mod. Phys., 17, 120

Einstein, A., \& Straus, E. G. 1946, Rev. Mod. Phys., 18, 148

El-Ad, H., \& Piran, T. 1997, ApJ, 491, 421

Faber, S. M, Courteau, S., Dekel, A., et al. 1994, J. Roy. Astron. Soc. Can., 88(2)

Fairall, A. 1997, Large-scale structure in the Universe (Wiley-Praxis) Fisher, K. B., Huchra, J. P., Strausss, M. A., et al. 1995, ApJS, 100, 69 Hoyle, F., \& Vogeley, M. S. 2002, ApJ, 566, 641

Kirshner R. P., Oemler, A., Schechter, P. L., \& Shectman, S. A. 1981, ApJ, 248, L57

Loeb, A. 1993, ApJ, 403, 542

Mukhanov, V. F., Feldman, H. A., \& Brandenberger, R. H. 1992, Phys. Rept., 215, 203

Oppenheimer, J. R., \& Snyder, H. 1939, Phys. Rev., 57, 455

Peebles, P. J. E. 2001, ApJ, 557, 495

Perlmutter, S., Aldering, G., Goldhaber, G., et al. 1999, ApJ, 517, 565

Rubano, C., \& Barrow, J. D. 2001, Phys. Rev. D, 64, 127301

Schimdt, B. P., Suntzeff, N. B., Phillips, M. M., et al. 1998, ApJ, 507, 46

Schmidt, J., Ryden, B. S., \& Mellott, A. 2001, ApJ, 546, 609

Schneider, P., Ehlers, J., \& Falco, E. E. 1992, Gravitational Lenses (Berlin: Springer-Verlag)

Stornaiolo, C. 2002, GRG, 34, 2089

Tegmark, M., Strauss, M., Blanton, M., et al., Cosmological parameters from SDSS and WMAP, PRD, in press [arXiv: astro-ph/0310723]

Zeldovich, Ya. B., \& Sazhin, M. V. 1987, Sov. Astron. Lett., 13, 145 